Anca Gheaus

\title{
Die normative Bedeutung der Schwangerschaft stellt Leihmutterschaftsverträge in Frage
}

Zusammenfassung: Gebärende Mütter haben in der Regel ein moralisches Recht, ihre Neugeborenen aufzuziehen. Dieses Recht gründet in der gegenseitigen Bindung, die während der Schwangerschaft zwischen der austragenden Mutter und dem Fötus entstanden ist. Diese Bindung ist zum Teil durch die Belastungen der Schwangerschaft entstanden und dient dem Interesse des Neugeborenen; aber auch die austragende Mutter hat ein starkes Interesse am Schutz dieser Bindung. Das Recht, das ausgetragene Kind aufzuziehen, kann aufgrund seiner moralischen Grundlagen nicht beliebig auf andere Personen übertragen werden, die als soziale Eltern des betreffenden Kindes fungieren wollen. Dies zeigt, dass Leihmutterschaftsverträge illegitim sind und daher als nichtig angesehen werden sollten. ${ }^{12}$

\section{Einleitung}

Eine Leihmutter (bzw. Surrogatmutter) ist eine Frau, die sich bereit erklärt, schwanger zu werden und dann ein Kind auszutragen und zur Welt zu bringen, für das andere als soziale Eltern fungieren werden. ${ }^{3}$ In manchen Fällen ist die Leihmutter auch die genetische Mutter. In anderen Fällen ist das Kind genetisch nicht mit der Leihmutter, aber mit einem oder beiden der vorgesehenen sozialen Eltern verwandt. Viele Rechtsordnungen gestatten derzeit Einzelpersonen den Abschluss kommerzieller Leihmutterschaftsverträge (wie z. B. Indien, Russland und einige der Vereinigten Staaten), während andere die Leihmutterschaftsvereinbarungen auf die so genannte altruistische Leihmutterschaft beschränken; d. h.: auf Fälle, in

1 Dieses Projekt wurde vom Europäischen Forschungsrat (ERC) im Rahmen des Forschungs- und Innovationsprogramms „Horizon 2020“ der Europäischen Union finanziert (Grant Agreement Number:: 648610).

2 Dieser Aufsatz wurde von Hannes Wendler ins Deutsche übersetzt und erschien zunächst in englischer Sprache unter Gheaus, Anca (2016): The normative importance of pregnancy challenges surrogacy contracts, in: Analize - Journal of Gender and Feminist Studies 6/20, 20-31. $3 \mathrm{Zu}$ einer Auseinandersetzung mit der Leihmutterschaft aus medizinischer und psychologischer Perspektive vgl. den Beitrag von Heribert Kentenich zum vorliegenden Band.

Ә OpenAccess. (C) 2021 Olivia Mitscherlich-Schoennherr, published by De Gruyter. (cc))BY-NC-ND This work is licensed under the Creative Commons Attribution 4.0 International License. 
denen die Leihmutter ein Kind für eine andere Person - in der Regel einen Verwandten - aus nichtkommerziellen Interessen austrägt. Wieder andere Rechtsordnungen (wie z.B. Deutschland oder Quebec in Kanada) verbieten jegliche Leihmutterschaft oder erklären sie zumindest für ungültig. Darüber hinaus stellt sich die Frage der Durchsetzbarkeit von Leihmutterschaftsverträgen: Einige Rechtsordnungen versuchen Leihmutterschaftsverträge nicht durchzusetzen, so dass es der Leihmutter rechtlich freisteht, das Kind zu behalten, sollte sie ihre Meinung ändern (womöglich nach Rückzahlung etwaiger Gebühren und schwangerschaftsbezogener Kosten, die das auftraggebende Paar bereits übernommen hatte).

Ob es überhaupt Leihmutterschaftsverträge geben sollte und, falls ja, ob sie durchsetzbar sein sollten oder nicht, wird offenbar viele Fragen aufwerfen. Bisher wurde in der Literatur zum normativen Status der Leihmutterschaft vor allem erörtert, ob die Leihmutterschaft an sich ausbeuterisch oder anderweitig schädlich für die Leihmutter ist, und ob die bestehenden Leihmutterschaftspraktiken tatsächlich ausbeuterisch oder anderweitig schädlich für die Leihmutter sind. Eine andere Reihe von ebenso wichtigen Fragen, die sich mit dieser Angelegenheit befassen, betreffen die Art und Weise, wie Individuen ein moralisches Recht auf die Erziehung eines Kindes erwerben, und ob sie dieses Recht, sobald sie es erworben haben, willkürlich auf andere Individuen übertragen können. ${ }^{4}$

Dieser Aufsatz geht auf die letztgenannte Gruppe von Fragen ein, um zur Debatte über die Legitimität von Leihmutterschaftsverträgen und ihre Durchsetzbarkeit beizutragen. Ich argumentiere, dass die Schwangerschaft normativ relevant ist für die Frage, wer das moralische Recht haben sollte, ein bestimmtes Kind aufzuziehen. Zumindest solange nicht andere Menschen mit hoher Wahrscheinlichkeit bessere Eltern für das betreffende Kind abgeben und gewillt sind, es $\mathrm{zu}$ erziehen, erwirbt eine austragende Mutter das moralische Recht auf Elternschaft vermöge der Tatsache, dass sie das Kind ausgetragt hat. Darüber hinaus sind die Gründe für das Innehaben dieses Rechts so beschaffen, dass dieses Recht nicht auf andere Personen übertragen werden kann. Das von der austragenden Mutter erworbene moralische Recht ist selbstredend anfechtbar. Wie ich erläutern werde, wird das moralische Recht nichtig, wenn die betreffende Frau für ihr Kind kein hinreichend gutes Elternteil ist; und das Recht besteht gar nicht erst - zumindest nicht vermöge der Schwangerschaft -, für den Fall, dass es keine

4 In diesem Aufsatz verwende ich das nicht weiter qualifizierte Wort „Recht“, um auf gesetzliche Rechte zu verweisen. Wenn ich mich auf moralische Rechte beziehe, mache ich es immer explizit und der Großteil der Diskussion hier handelt von moralischen Rechten. Ich gehe davon aus, dass die Existenz eines moralischen Rechts auf $\mathrm{x}$ eine wichtige Grundlage für ein gesetzliches Recht auf $\mathrm{x}$ sein sollte, dass aber die Übersetzung nicht immer direkt sein muss. 
gegenseitige Bindung zwischen der Mutter und dem Neugeborenen gibt. ${ }^{5}$ Wenn Leihmütter aufgrund einer während der Schwangerschaft entstandenen Bindung ein moralisches Recht erwerben, die Kinder zu erziehen, dann sollten Leihmutterschaftsverträge nicht durchsetzbar sein, weil ihre Durchsetzung ein moralisches Recht der Leihmutter verletzen würde. Und wenn ein Teil der Erklärung für dieses moralische Recht die Bindung des Neugeborenen an ihre oder seine austragende Mutter ist, dann kann das moralische Elternrecht der Leihmutter prinzipiell nicht auf einen Dritten übertragen werden, weshalb Leihmutterschaftsverträge immer als nichtig angesehen werden sollten.

Einige Leihmütter weigern sich in der Tat, das von ihnen ausgetragene Kind abzugeben. Wenn das Paar, das die Leihmutter angeworben hatte, weiterhin wünscht, die sozialen Eltern des Kindes zu werden, wird es wahrscheinlich zu Sorgerechtsstreitigkeiten kommen, und verschiedene Rechtsordnungen werden in solchen Fällen unterschiedlich entscheiden. Manchmal ist das Gesetz auf der Seite der Leihmutter, indem es sie als rechtliche Mutter des Kindes anerkennt (was wiederum das Sorgerecht zur Folge haben kann). Aber in verschiedenen Rechtsordnungen kann der Grund für die Zuerkennung des Sorgerechts an die Leihmutter sehr unterschiedlich sein.

1988 wurde in einem der ersten Leihmutterschaftsfälle, die in den Vereinigten Staaten vor Gericht gebracht wurden, die Leihmutter Mary Beth Whitehead schließlich als gesetzliche Mutter des von ihr ausgetragenen Babys („Baby M“) anerkannt, nachdem sie von dem Ehepaar William und Elizabeth Stern angeworben worden war (Sanger 2007). Baby M wurde durch künstliche Befruchtung gezeugt, und William Stern war ihr genetischer Vater. (Das Sorgerecht wurde letztlich dem Vater, William Stern, auf der Grundlage des Prinzips des Kindeswohls gewährt.) Wichtig ist in diesem Fall, dass Mary Beth Whitehead sowohl Eizellspenderin als auch austragende Mutter war. Ihre genetische Verwandtschaft mit dem Kind dürfte die Entscheidung beeinflusst haben. In der Tat ist in einigen Rechtsordnungen das wichtigste Kriterium, das zur Entscheidung von Streitigkeiten über den elterlichen Status herangezogen wird, die genetische Verwandtschaft. ${ }^{6}$

In anderen Fällen muss die genetische Verwandtschaft jedoch keine Rolle bei der Entscheidung über die Gewährung von Elternrechten an die Leihmutter

5 Es mag andere Fälle geben, in denen die austragende Mutter unter Berücksichtigung aller Umstände nicht das moralische Recht hat, das Kind, das sie ausgetragen hat, großzuziehen. Andernorts (Gheaus 2015b) erörtere ich die Möglichkeit, dass das moralische Recht, ein bestimmtes Kind großzuziehen, dem besten verfügbaren Elter zusteht (und wie sich dies zum Austragen eines Kindes verhält).

6 Für eine philosophische Analyse und Verteidigung hiervon siehe Richards (2010). 
spielen. In einem jüngeren Fall aus dem Vereinigten Königreich hat eine Leihmutter, die ein Kind für ein Paar zur Welt gebracht hatte und ihre Meinung bezüglich des Verzichts auf das Kind geändert hatte, das Sorgerecht aufgrund der Bindung erhalten, die während der Schwangerschaft zwischen der werdenden Mutter und ihrem Neugeborenen entstanden ist. Mit den Worten von Richter Baker, der den Fall entschieden hat:

„[T]here is a clear attachment between mother and daughter. To remove her from her mother's care would cause a measure of harm. The natural process of carrying and giving birth to a baby creates an attachment which may be so strong that the surrogate mother finds herself unable to give up the child.“ (Baker 2011)

Eben diese Unfähigkeit - oder, was wahrscheinlicher ist: der Unwille - der Leihmutter, das Kind aufzugeben, ist Teil dessen, was es für die Leihmutter wahrscheinlicher macht (unter sonst gleichen Bedingungen), bestmöglich für die emotionalen Bedürfnisse des Kindes zu sorgen als Dritte.

Ich nehme an, dass die Argumentation von Richter Baker stichhaltig ist - und die folgende Analyse der normativen Bedeutung der Schwangerschaft soll dies entfalten.

\section{Wie Schwangerschaft normativ bedeutsam ist - ein phänomenologischer Ansatz ${ }^{7}$}

Die Mehrheit der Schwangerschaften weist zwei allgemeine Merkmale auf, die für ein moralisches Recht von potentiell geeigneten Müttern sprechen, ihre Neugeborenen zu behalten und aufzuziehen. Erstens verursachen Schwangerschaften eine Vielzahl von Kosten: physische, psychische, soziale und finanzielle. Die meisten dieser Kosten können nur von schwangeren Frauen und bis zu einem gewissen Grad von dem sie unterstützenden Partner getragen werden, falls sie in einer Paarbeziehung leben. In diesem Zusammenhang bilden zweitens viele, möglicherweise die meisten, werdenden Mütter während der Schwangerschaft eine körper-leibliche, aber auch emotionale, intime Beziehung zu ihrem Fötus aus. $^{8}$

7 Dieser Abschnitt baut grundlegend auf Gheaus (2012) auf.

8 Ein streng physikalistischer Ansatz würde die Bindung zwischen der schwangeren Frau und dem Fötus auf Oxytocin zurückführen: einer Substanz, die während der Schwangerschaft ausgeschüttet wird. Ob man zur Analyse der Bindung während der Schwangerschaft den phänomenologischen oder den physikalistischen Ansatz wählt, mag normativ einen Unterschied ma- 
Die beiden hier diskutierten Merkmale der Schwangerschaft können - zusammen mit anderen Bedingungen ${ }^{9}$ - ein moralisches Recht begründen, das selbstgeborene Kind $\mathrm{zu}$ behalten: ein Recht, das gleichzeitig im Interesse der Eltern und im Interesse des Kindes begründet ist.

Arbeiten zur Phänomenologie der Schwangerschaft sind sehr hilfreich für das Verständnis beider Merkmale. In der Auseinandersetzung mit beiden Merkmalen stütze ich mich auf die Arbeit mehrerer Feministinnen und beziehe mich dabei insbesondere auf Amy Mullins Buch „Reconceiving Pregnancy and Childcare“.

Die Kosten einer Schwangerschaft sind vielfältig: körper-leiblich, emotional, sozial und finanziell. Sie bestehen in den Schmerzen beim Gebären und Entbinden, in der eingeschränkten Autonomie der Schwangeren, in den Gesundheitsrisiken, die Frauen eingehen, um ihr Kind auszutragen, in den Sorgen um die Gesundheit von Mutter und Kind und in der beängstigenden Gefahr einer Fehlgeburt. $^{10}$

Die körper-leiblichen Belastungen der Schwangerschaft haben erhebliche Auswirkungen auf die Fähigkeit vieler schwangerer Frauen, ihr Leben wie gewohnt weiterzuführen:

„Fatigue, high blood pressure, excessive water retention in one's hands and feet, nausea and vomiting, an inability to carry heavy objects, and other common symptoms of pregnancy do involve suffering and affect a pregnant woman's ability to carry out her daily tasks, whether in paid employment, domestic work, childcare or interactions with friends and family, regardless of how accommodating her environment may be.“ (Mullin 2005, 64)

Einige der wichtigsten Belastungen der Schwangerschaft ergeben sich aus dem Ausmaß und dem Tempo der Veränderungen, die schwangere Frauen durchmachen. Mullins Buch vermittelt einen sehr lebhaften Eindruck von den vielen körper-leiblichen Veränderungen, die schwangere Frauen ,in visual acuity, pigment of her skin, the onset of rashes, nausea, heartburn, raised blood pressure, increased congestion, difficulty catching her breath, swollen hands and feet“

chen, aber nicht in diesem Zusammenhang. Eine Physikalistin bzw. ein Physikalist könnte zum Beispiel argumentieren, dass wir Oxytocin einsetzen könnten und in manchen Kontexten auch sollten, um die emotionale Beziehung zwischen nicht-leiblichen Eltern und Babys zu fördern. Aber dies würde das vorliegende Argument nicht betreffen, das besagt, dass die Schwangerschaft selbst diese Beziehung auf die eine oder andere Weise fördert und dass es daher moralisch falsch ist, Babys ihren leiblichen Eltern wegzunehmen.

9 Ich meine, kann dies hier aber nicht vollständig ausführen, dass diese Bedingungen damit zu tun haben, dass die in Frage kommende Mutter bestimmte Kriterien der elterlichen Eignung erfüllt.

$10 \mathrm{Zu}$ den schwerwiegenden Leiden, die eine Fehlgeburt für die schwangere Frau mit sich bringen kann, siehe Cahill/Norlock/Stoyles (2015). 
(Mullin 2005, 39) durchmachen. Viele davon sind relativ geringfügig, aber zusammen können sie erhebliche Schmerzen und Störungen des normalen Lebens nach sich ziehen. Diese Veränderungen werden von der überwiegenden Mehrheit der schwangeren Frauen erlebt und tragen oft zu einem ausgeprägten Gefühl des Kontrollverlustes über das eigene Leben und zu einer verminderten Fähigkeit bei, während der Schwangerschaft und während der Erholung von der Geburt anderen Projekten und Interessen nachzugehen. Wie Mullin anmerkt, ,at no other time will an otherwise healthy adult undergo such widespread, rapid and undesired change in the shape and size of her body, in the way she moves, eats and sleeps.“ (Mullin 2005, 67).

Viele schwangere Frauen zahlen auch ,Verhaltenskosten' mit der Einschränkung dessen, was sie essen und trinken, welche Drogen sie konsumieren und welchem Sport und sonstigen körperlichen Aktivitäten sie nachgehen können. Es gibt soziale Kosten zu tragen, wie z.B. Bevormundung und ungebetene Vertraulichkeit: Schwangeren Frauen wird oft gesagt ,that nothing they can do could be more important than their job of bringing a child to life" (Mullin 2005, 40). Im Allgemeinen verändert die Schwangerschaft die Beziehungen der werdenden Eltern zu ihrer unmittelbaren Familie, zu Freund_innen, Kolleg_innen und, wenn die Schwangerschaft sichtbar ist, sogar zu Fremden, auf unkontrollierbare Weisen. Es zählen wohl nicht alle dieser Veränderungen als Kosten, da in einigen Fällen schwangere Frauen willkommen geheißene Beziehungen entwickeln oder stärken, die z.B. auf Verbundenheit mit anderen Eltern beruhen. Aber viele der Veränderungen sind nicht wünschenswert und, was für das vorliegende Argument wichtig ist, viele der wünschenswerten Veränderungen basieren auf der Annahme, dass die schwangeren Frauen ihr leibliches Kind weiterhin erziehen werden. Darüber hinaus wären die Kosten der Schwangerschaft tatsächlich höher, wenn die gebärenden Mütter nicht wüssten, ob sie berechtigt sein werden, ihr leibliches Kind zu behalten. Ein häufig gegebener Trost für schwangere Frauen ist, dass die Elternschaft alle Mühen und Schmerzen wert ist.

Schließlich müssen werdende Mütter spezifische emotionale Belastungen auf sich nehmen, wie die Furcht vor einer Fehlgeburt oder die Furcht, über die Fortsetzung einer Schwangerschaft mit erheblichen gesundheitlichen Risiken entscheiden zu müssen. Eine schwangere Frau „needs to come to terms with her welcoming of a creature who is already transforming her body, her social interactions, and her habits, who will always radically transform her life and about whom she knows virtually nothing“ (Mullin 2005, 43). Obgleich sie nicht alle diese Kosten übernehmen können, können die involvierten Partner in der Regel viele mittragen. Sie sind oft die Hauptquelle der emotionalen, praktischen und finanziellen Unterstützung ihrer schwangeren Partnerin: Sie können sie zu Arztbesuchen begleiten und sie während der Geburt unterstützen, sie können ihre Sorgen 
teilen und versuchen, sie zu mildern, sie von einem Teil ihrer regulären Arbeit entlasten und als Schnittstelle zwischen ihr und der nicht ausreichend entgegenkommenden Außenwelt dienen. Einige, aber nicht alle Kosten einer Schwangerschaft können sozial verhindert oder gemildert werden - darauf gehe ich im Folgenden näher ein.

Eine Schwangerschaft umfasst häufig neben den Kosten auch spezifische Vorzüge. Die Vorzüge und Freuden einer Schwangerschaft heben die Kosten jedoch nicht auf und machen die Schwangerschaft nicht $\mathrm{zu}$ einer intrinsisch wünschenswerten Erfahrung. Bestimmte Vorteile der Schwangerschaft, wie z.B. die erhöhte Aufmerksamkeit und Fürsorge, die schwangere Frauen häufig erhalten, sollen ihre Kosten abmildern: Wenn die Schwangerschaft nicht mit spezifischen Kosten verbunden wäre, würde es auch diese Vorteile nicht geben (in dieser Hinsicht ist die Schwangerschaft mit Krankheit und Behinderung vergleichbar). Andere Vorzüge der Schwangerschaft, wie z. B. die freudige Erwartung des Babys, sind nur dann wertvoll, wenn die Schwangere davon ausgeht, dass sie das Kind, das sie in sich trägt, behalten und aufziehen wird. Wichtig ist, dass die salientesten positiven Aspekte der Schwangerschaft von der Erwartung abhängen, am Ende der Schwangerschaft Mutter zu werden. Wenn sich entgegen den Behauptungen, die ich in diesem Abschnitt vorbringe, die Schwangerschaft als intrinsisch wertvoll erwiese, oder wenn die Vorzüge der Schwangerschaft ihre Belastungen überwögen, ohne von der Erwartung abzuhängen, das Baby zu behalten, dann würde eine Säule meines Arguments für ein moralisches Recht einbrechen, das ausgetragene Kind aufzuziehen.

Bestimmte Schwangerschaftserfahrungen, wie z. B. Schlafprobleme und unterbrochene Lebensgewohnheiten, können gerade aufgrund ihrer Entbehrungen werdende Eltern darauf vorbereiten, sich besser um ihre Babys zu kümmern. Einige Autor_innen sind der Meinung, dass die Schwangerschaft dazu beiträgt, die Mütter - und, wenn sie eng mit der Schwangerschaft verbunden sind, auch ihre Partner - auf die großen Veränderungen vorzubereiten, die die Kindererziehung mit sich bringt (Levesque-Lopman 1983, 256). Wenn dies richtig ist, dann bedeutet es ceteri paribus, dass leibliche Mütter besser darauf vorbereitet sind, sich um ein Baby zu kümmern, sodass ein Interesse des Kindes daran besteht, von einer Frau aufgezogen zu werden, die auch eine leibliche Mutter ist. Die Belastungen der Schwangerschaft führen dann zu einem auf dem Kindeswohl beruhendem moralischen Recht, bestimmte Babys zu erziehen.

Es besteht eine gewisse Ähnlichkeit zwischen der Rechtfertigung eines moralischen Rechts auf Elternschaft durch die Berufung auf die Kosten einer Schwangerschaft und dem libertären - oder proprietären - Argument, wonach Eltern eine Art Eigentumsanspruch an Kindern haben, weil Kinder aus der Arbeit ihrer Eltern resultieren (Narveson 2002). Meinerseits trete ich jedoch nicht dafür 
ein, dass die Kosten der Schwangerschaft, einschließlich der dafür erforderlichen Anstrengungen, einen starken eigentumsähnlichen Anspruchmit sich bringen.

Das Argument der Schwangerschaftskosten könnte eine gewisse Rechtfertigung für ein moralisches Recht darstellen, das ausgetragene Kind zu behalten und großzuziehen; es könnte unter Umständen aber hinter andere Gründe zurücktreten - etwa Erwägungen bezüglich ,race'- oder Geschlechtergerechtigkeit. ${ }^{11}$ Die Berufung auf die Kosten der Schwangerschaft allein kann legitimes ,Babytauschen' zwischen allen austragenden Müttern nicht ausschließen. Die Belastungen der Schwangerschaft berechtigen potenziell geeignete Eltern, die gerade entbunden haben, ein Baby zu erziehen, aber nicht notwendigerweise das bestimmte Kind aufzuziehen, das sie geboren haben. Ebenso ist darauf hinzuweisen, dass die alleinige Berufung auf die Kosten der Schwangerschaft ein moralisches Recht auf Erziehung begründen würde, das seinerseits leicht auf Dritte übertragbar wäre und damit zugleich Leihmutterschaftsverträge legitimieren könnte. Wenn austragende Mütter das moralische Recht, ihr Neugeborenes zu erziehen, bloß aus den Schwangerschaftskosten erlangten - womöglich dank der Arbeit, die sie in diesen Prozess gesteckt haben -, gäbe es keinen offensichtlichen Grund, der dagegen spräche, dieses Recht anderen Personen zu verkaufen.

Das zweite Merkmal der Schwangerschaft leistet den Großteil der argumentativen Arbeit: die Tatsache, dass die Schwangerschaft das Entstehen einer intimen Beziehung zwischen der gebärenden Mutter und dem zukünftigen Kind erleichtert. Die beiden Merkmale der Schwangerschaft - ihre erheblichen Kosten und der durch sie ermöglichte Aufbau einer intimen Beziehung mit dem zukünftigen Baby - sind eng miteinander verzahnt. Mit der Schwangerschaft müssen schwangere Frauen - und manchmal ihre unterstützenden Partner - erhebliche Ressourcen investieren, um Kinder zur Welt zu bringen; dies ist oft ein bewusster, absichtlicher Prozess, ähnlich wie bei anderen Projekten, die Menschen verfolgen: Mit ihnen sind viel Antizipation und Planung, Denken und Hoffen, Imagination und Projektion verbunden. Zugegeben, im Falle von Leihmüttern können einige dieser psychologischen Prozesse fehlen: Wenn die werdende Mutter nicht erwartet, das Kind zu erziehen, kann sie gezielt versuchen, sich nicht auf Vorfreude und Planen, Denken und Hoffen einzulassen. Es ist jedoch nicht klar, ob es möglich ist, ein solches Engagement zu vermeiden; auch Leihmütter können -

11 Bei der Beantwortung der Frage, wer das moralische Recht hat, ein bestimmtes Kind zu erziehen, müssen alle relevanten Aspekte berücksichtigt werden. Für Argumente zugunsten der „Babylotterie“, siehe Gheaus (2012) und Earl (2015), Manuskript. 
wenngleich unfreiwillig und möglicherweise unbewusst - Antizipation, Hoffnung und Projektion erleben.

Durch ihre körper-leibliche Verbindung mit dem Baby und ihre vielfältigen psychologischen Investitionen bauen schwangere Frauen normalerweise eine Beziehung zu ihrem zukünftigen Baby auf: eine Beziehung, die manchmal hochemotional ist und bereits bei der Geburt recht weit entwickelt ist. Die gebärende Mutter und ihre Neugeborenen haben bereits eine gemeinsame Geschichte einschließlich zahlreicher leiblich geteilter Erfahrungen („Du hast mich am ersten März getreten“, „Du hast mir Sorgen bereitet“, „Du hast mich vor Glück weinen lassen“). Die Schwangerschaft formt damit einen Prozess, in dessen Verlauf sich eine zugleich körper-leibliche und emotionale Bindung der Schwangeren an das Kind in besonderem Maße entwickeln kann. Caroline Whitbeck ist so weit gegangen, dafür einzutreten, dass die Zuneigung zu eigenen Kindern, die oft mit Bezug auf einen mütterlichen/elterlichen 'Instinkt' erklärt wird, eigentlich auf leiblichen Erfahrungen basiert: „parental affection or attachment is influenced by experience, and this experience is not confined to socialization experience but includes, in a large measure, bodily experiences that are the same cross-culturally; i.e. all women have special bodily experiences that are likely to enhance those feelings, attitudes and fantasies, which induce people to generally care for their infants“ (Whitbeck 1984, 191). Diese Erfahrungen umfassen Schwangerschaft, Wehen, Geburt und postnatale Erholung, welche allesamt einzigartig für die gebärende Mutter sind. Whitbeck kommt zu dem Schluss, dass, obwohl der mütterliche ,Instinkt‘ selbst ein Mythos ist, die Biologie - durch leiblich geteilte Erfahrung - eine sehr wichtige Rolle beim Bindungsaufbau zwischen Neugeborenen und ihren Müttern spielt. Dieses Argument könnte bald überprüfbar sein, wenn es genügend Menschen gibt, deren austragende Mütter sich von ihren genetischen Müttern unterscheiden. Wenn Whitbecks Argument stimmt, dann verweist es auf die wichtige Rolle, die die Biologie in Eltern-KindBeziehungen dank der körper-leiblichen Prozesse der Elternschaft und unabhängig von genetischen Verbindungen spielt.

Wie bei der Übernahme der Schwangerschaftskosten können die begleitenden Partner während der Schwangerschaft direkt am Prozess der Herausbildung der Beziehung der Schwangeren zum Baby teilnehmen. Mit Hilfe der Medizintechnik können sie - genauso früh wie die werdende Mutter - den Fötus sehen und seinen Herzschlag hören; in den letzten Phasen der Schwangerschaft können sie das Baby fühlen, mit ihm sprechen und von ihm gehört werden. Genau wie die Mutter können sie die Ängste, Hoffnungen und Phantasien erleben, die der heranwachsende Fötus auslöst.

Die Phänomenologie der Schwangerschaft zeigt nicht, dass alle Schwangerschaften zu intimen Beziehungen zwischen gebärenden Müttern und ihren Neu- 
geborenen führen. Sie zeigt nur, dass eine Schwangerschaft zu einer Bindung führen kann und wahrscheinlich auch führen wird; die Wahrscheinlichkeit ist dabei sehr groß, denn eine Bindung kann auch dann entstehen, wenn die Schwangere weiß, dass sie das Kind nicht behalten darf, wie wir aus Fällen von Leihmüttern wissen, die eine starke Bindung $\mathrm{zu}$ ihrem ungeborenen und dann neugeborenen Kind entwickelt haben. Die Bindung während der Schwangerschaft liefert einen überaus belastbaren Grund, weshalb die Weitergabe von Babys an Dritte als soziale Eltern wahrscheinlich bereits bestehende, intime Beziehungen zwischen Neugeborenen und ihren austragenden Eltern zerstören würde.

Die Tatsache, dass die Beziehung zum eigenen, zukünftigen Kind während der Schwangerschaft beginnt, stellt den fehlenden Schritt in der Rechtfertigung dar, die einige Philosoph_innen zugunsten des moralischen Rechts entwickelt haben, das gezeugte Kind zu behalten und aufzuziehen. Dieselbe Bindung, die im Zusammenhang mit der Schwangerschaft entstanden ist, bietet eine Antwort auf die allgemeinere Frage danach, wie man das moralische Recht bestimmt, ein bestimmtes Kind zu erziehen. (Die Antwort ist allgemein, aber nicht universell: In einigen Fällen stirbt die werdende Mutter bei der Geburt oder ist nicht willens, ihr moralisches Recht auszuüben, das von ihr geborene Kind aufzuziehen. In diesen Fällen werden andere Erwägungen darüber entscheiden, wer das moralische Recht hat, das Kind aufzuziehen.) Einige Philosoph_innen - vor allem Ferdinand Shoeman (1980) sowie Harry Brighouse und Adam Swift (2006; 2014) -, haben auf die Unzulässigkeit verwiesen, bereits etablierte intime Beziehungen zwischen Eltern und Kindern zu stören, um dafür einzutreten, dass alle zur Elternschaft geeigneten Erwachsenen ein moralisches Recht hätten, soziale Eltern der betreffenden Kinder zu werden. Die Gründe dafür liegen sowohl im Wohlergehen der Eltern als auch der Kinder. Es wurde jedoch kein Grund angegeben, warum sich solche Beziehungen überhaupt erst haben entwickeln dürfen - vor allem: wenn andere Personen ebenfalls dazu bereit gewesen wären, als soziale Eltern der betreffenden Kinder zu fungieren. Mein Ansatz zum normativen Wert der Schwangerschaft füllt diese Lücke: Wenn derselbe Prozess, der Babys auf die Welt bringt, auch deren erste intimen Beziehungen zu Erwachsenen mit sich bringt, bedürfen die Beziehungen zwischen leiblichen Eltern und ihren Babys keiner Rechtfertigung: Sie bestehen von Anfang an.

Es ist wichtig, zu dieser Analyse die Perspektive des Babys hinzuzufügen, das sich in der Regel auch an seine Mutter bindet: deren Stimme, Herzschlag usw. es in der letzten Phase der Schwangerschaft erkennen kann (DeCasper/Fifer 1980; Beauchemin et al. 2011). ${ }^{12}$ Dass herkömmlicherweise auch das Neugeborene an

12 Ich bin Jake Earl dankbar dafür, mich auf diese Artikel aufmerksam gemacht zu haben. 
die werdende Mutter gebunden ist - soweit wir das beurteilen können, und soweit von einem Neugeborenen ausgesagt werden kann, dass es gebunden ist -, liefert eine zusätzliche, kindzentrierte Rechtfertigung für das moralische Recht, das ausgetragene Kind $\mathrm{zu}$ erziehen.

Zusammenfassend ist die besondere Art und Weise, in der wir zur Welt kommen, wesentlich, um zu bestimmen, wer das moralische Recht hat, uns aufzuziehen. Wenn wir alle in Laboratorien zur Welt kämen, geschaffen von Wissenschaftler_innen, gäbe es wenig Grund, den Menschen, die das genetische Material zur Verfügung gestellt haben, ein Recht auf unsere Erziehung einzuräumen. ${ }^{13}$ In der Tat habe ich andernorts dafür argumentiert, dass es Gerechtigkeitsgründe für eine Umverteilung von Babys zwischen allen potenziellen, adäquaten Eltern gibt, und in bestimmten sozialen Kontexten würde ein ,Babytauschen' dazu beitragen, historische und tief verwurzelte Assoziationen zwischen ,race' oder Geschlecht und Bevorteilung anzugehen (Gheaus 2012).

Um dieser Herausforderung zu begegnen, habe ich einen Ansatz dafür entwickelt, wie Menschen das Recht erwerben, ein bestimmtes Baby zu erziehen. Ein Element eines derartigen Rechtserwerbs besteht im moralischen Recht, das ausgetragene Kind zu behalten und aufzuziehen. Wenn zum Zeitpunkt der Geburt geeignete, austragende Mütter (und manchmal auch ihre beteiligten Partner_innen) bereits beträchtliche Kosten dafür bezahlt haben, Eltern zu werden, und zum Teil aufgrund dieses Prozesses eine erste intime Beziehung zu dem Kind entwickelt haben, dann sind sie mehr als andere, gleichermaßen geeignete, potenzielle Eltern berechtigt, das Kind, das sie geboren haben, zu erziehen. Dieser Unterschied zwischen geeigneten, austragenden Müttern und anderen geeigneten, potentiellen Eltern kann die notwendige Rechtfertigung dafür liefern, ein fundamentales moralisches Recht auf Elternschaft im Allgemeinen (wie es von Shoeman, Brighouse und Swift verteidigt wird) in ein moralisches Recht austragender Mütter auf die Elternschaft ihres geborenen Kindes zu übersetzen.

\section{Konklusionen für Leihmutterschaftsverträge}

Wie genau Erwachsene das moralische Recht erwerben können, ein bestimmtes Kind zu erziehen, ist eine kontroverse Frage, die mit diesem kurzen Artikel nicht geklärt werden sollte. ${ }^{14} \mathrm{Zu}$ den verfügbaren Optionen gehören die Berufung auf

13 Hierfür argumentiere ich andernorts (Gheaus 2015a) ausführlich.

14 Für eine diesbezüglich hilfreiche Diskussion siehe Swift und Brighthouse (2014). 
die Interessen des Kindes, die Berufung auf die Interessen der Erwachsenen, die soziale Eltern werden wollen, und die Berufung auf beide Arten von Interessen. Hier habe ich argumentiert, dass die mit der Schwangerschaft einhergehenden Tatsachen - d.h. die ihr inhärenten Kosten und die hohe Wahrscheinlichkeit, dass die Schwangerschaft ein Prozess darstellt, in dessen Verlauf die austragende Mutter und ihr Fötus eine gegenseitige Bindung formen - darauf hindeuten, dass austragende Mütter sehr wahrscheinlich ein pro tanto moralisches Recht auf die Elternschaft ihrer Neugeborenen haben. Dabei kann eine Vielzahl von Ansätzen darüber berücksichtigt werden, wie man das moralische Recht auf Elternschaft erwirbt. (Eine bemerkenswerte Ausnahme ist der Ansatz, nach dem es der Beitrag des eigenen genetischen Materials ist, der das moralische Recht auf Elternschaft begründet. Dieser Ansatz ist sehr einflussreich - sowohl in der Populärmoral als auch in der Rechtspraxis - allerdings, wie ich meine, auch sehr unplausibel. ${ }^{15}$

Sicherlich haben austragende Mütter nicht immer das moralische Recht, ihr leibliches Kind zu erziehen, weil die Schwangerschaft selbst nicht das Recht dazu verleiht: Vielmehr erhalten austragende Mütter das Recht aufgrund der Bindung, die sie gewöhnlich mit dem Kind eingehen, und diese Bindung besteht nicht immer. Darüber hinaus können andere Umstände das moralische Recht nichtig werden lassen - wie z.B. in Fällen, in denen die austragende Mutter kein ausreichend geeignetes Elternteil abgäbe.

Aber das Recht ist notwendigerweise ein ,stumpfes Instrument', das nicht alle normativ relevanten Merkmale jedes Falles berücksichtigen kann, den es abdeckt. Wenn das Recht einer austragenden Mutter in der Regel nicht unterlaufen wird, dann sollten Leihmutterschaftsverträge nicht gegen den Wunsch der austragenden Mutter, das Kind selbst zu erziehen, durchgesetzt werden. (Es sei denn, dass besondere Umstände - wie die oben genannten - vorliegen.) Da das moralische Recht zudem aufgrund einer gegenseitigen Bindung besteht und da diese Bindung dem Interesse des Neugeborenen dient, kann das Recht nicht nach Belieben auf andere Personen übertragen werden, die als soziale Eltern des betreffenden Kindes fungieren möchten. Dies weist darauf hin, dass Leihmutterschaftsverträge illegitim und daher nichtig sind.

\section{Literatur}

Baker, Jonathan (2011): CW v NT and another [2011] EWHC 33, Family Law Week, 2011 Archiv. Online verfügbar unter: http://www.familylawweek.co.uk/site.aspx?i=ed79071 (Zuletzt abgerufen: 05. 04.2016).

15 Hierfür argumentiere ich andernorts (Gheaus 2015a) ausführlich. 
Beauchemin, Maude et al. (2011): Mother and Stranger: An Electrophysiological Study of Voice Processing in Newborns, in: Cerebral Cortex 21/8, 1705-1711.

Brighouse, Harry/Swift, Adam (2006): Parents' Rights and the Value of the Family, in: Ethics 117/1, 80-108.

Brighouse, Harry/ Swift, Adam (2014): Family Values. The Ethics of Parent-Child Relationships, Princeton.

Cahill, Ann J/Norlock, Kathryn J./Stoyles, Byron J. (2015): Miscarriage, Reproductive Loss, and Fetal Death, in: Journal of Social Philosophy 46, 2015.

DeCasper, Anthony J./Fifer, William P. (1980): Of Human Bonding: Newborns Prefer Their Mothers' Voices, in: Science 208 (4448), 1174-1176.

Earl, Jake (2015): The Baby Lottery (Konferenzbeitrag in: Close Personal Relationships, Children and the Family, Universität Umea, 9-10.09.2015).

Gheaus, Anca (2012): The right to parent one's biological baby, in: Journal of Political Philosophy 20, $432-455$.

Gheaus, Anca (2015a): Biological parenthood: gestational not genetic (Workshopbeitrag in: MANCEPT workshop series, Universität Manchester, 02.2015.)

Gheaus, Anca (2015b): The best available parent (Konferenzbeitrag in: Close Personal Relationships, Children and the Family, Universität Umea, 9-10.09.2015).

Kentenich, Heribert: „Leihmutterschaft ist mit der Menschenwürde nicht vereinbar“. Anmerkungen zum Statement der Bundesregierung. Medizinische und psychologische Überlegungen, im vorliegenden Band.

Levesque-Lopman, Louise (1983): Decision and experience: a phenomenological analysis of pregnancy and childbirth, in: Human Studies 6, 247-277.

Mullin, Amy (2005): Reconceiving Pregnancy and Childcare. Ethics, Experience and Reproductive Labor, Cambridge.

Narveson, Jan (2002): Respecting Persons in Theory and Practice, Lanham, MD.

Richards, Norvin (2010): The Ethics of Parenthood, Oxford.

Sanger, Carol (2007): Developing markets in baby-making, in: Harvard Journal of Law and Gender 30, 67-97.

Shoeman, Ferdinand (1980): Rights of children, rights of parents and the moral basis of the family, in: Ethics 91/1, 6-19.

Whitbeck, Caroline: (1984): The Maternal Instinct, in: Treblicot, Joyce (Hg.): Mothering: Essays in Feminist Theory, Totowa, NJ, 185-191. 
